Georgian Mathematical Journal

Volume 14 (2007), Number 4, 651-659

\title{
RATE OF CONVERGENCE FOR THE BÉZIER VARIANT OF THE MKZD OPERATORS
}

\author{
VIJAY GUPTA AND HARUN KARSLI
}

\begin{abstract}
We estimate the rate of convergence of the Bézier variant of Durrmeyer type Meyer-König and Zeller operators for functions with derivatives of bounded variation defined on $[0,1]$.
\end{abstract}

2000 Mathematics Subject Classification: 41A25, 41A36.

Key words and phrases: Rate of convergence, approximation, MKZD operators, bounded variation.

\section{INTRODUCTION}

For a function $f$ defined on the interval $[0,1]$, the Meyer-König and Zeller (MKZ) operators $\tilde{M}_{n}(f, x)[13]$ are defined as

$$
\tilde{M}_{n}(f ; x)=\sum_{k=0}^{\infty} m_{n, k}(x) f\left(\frac{k}{n+k}\right),
$$

where $m_{n, k}(x)=\left(\begin{array}{c}n+k-1 \\ k\end{array}\right) x^{k}(1-x)^{n}$. To approximate Lebesgue integrable functions on the interval $[0,1]$, Guo [6] introduced the integrated MKZ operators

$$
\hat{M}_{n}(f ; x)=\sum_{k=0}^{\infty} \hat{m}_{n, k}(x) \int_{I_{k}} f(t) d t
$$

where $I_{k}=\left[\frac{k}{n+k}, \frac{k+1}{n+k+1}\right]$ and $\hat{m}_{n, k}(x)=(n+1)\left({ }_{k}^{n+k+1}\right) x^{k}(1-x)^{n}$. For the rate of convergence of some integral modifications of the MKZ operator we refer the reader to [5], [7] and [11]. In [15], Zeng defined, for each $\alpha \geq 1$, Bézier variants of the MKZ operators (1) and (2) by

$$
\tilde{M}_{n, \alpha}(f ; x)=\sum_{k=0}^{\infty} Q_{n, k}^{\alpha}(x) f\left(\frac{k}{n+k}\right)
$$

and

$$
\hat{M}_{n, \alpha}(f ; x)=\sum_{k=0}^{\infty}\left(\frac{Q_{n, k}^{\alpha}(x)}{\int_{I_{k}} d t}\right) \int_{I_{k}} f(t) d t,
$$

where $Q_{n, k}^{(\alpha)}(x)=\left(J_{n, k}(x)\right)^{\alpha}-\left(J_{n, k+1}(x)\right)^{\alpha}$ and $J_{n, k}(x)=\sum_{j=k}^{\infty} m_{n, j}(x)$ be the Bézier basis functions, which were introduced by P. Bézier [1]. In particular when $\alpha=1$, the operators (3) and (4) reduce to the operators (1) and (2), respectively. 
Very recently for $f \in L_{1}[0,1]$ and $\alpha \geq 1$, Gupta [8] introduced a Bézier variant of the different Durrmeyer type MKZ operators (MKZD) by

$$
M_{n, \alpha}(f ; x)=\sum_{k=0}^{\infty} Q_{n, k}^{\alpha}(x) \int_{0}^{1} b_{n, k}(t) f(t) d t, \quad x \in[0,1],
$$

where $b_{n, k}(t)=n\left(\begin{array}{c}n+k \\ k\end{array}\right) t^{k}(1-t)^{n-1}$. Gupta [8] investigated the rate of convergence of the operators (5), for functions of bounded variation on $[0,1]$ (see also [9]).

The aim of this paper is to extend the study on the operators (5) for functions having derivatives of bounded variation on $[0,1]$. Here we establish the rate of convergence of operators $M_{n, \alpha}$ for functions with derivatives of bounded variation defined on $[0,1]$. Several researchers have studied on MKZ operators and its different variants. We also mention the work on similar type of operators due to Bojanic and Cheng (see [2], [3]) who estimated the rate of convergence with derivatives of bounded variation for Bernstein and Hermite-Fejer polynomials by using different methods. Some of the important papers on this topic are due to Bojanic and Khan [4], Pych-Taberska [14], and Gupta et al. [10], [12].

Let $D B V[0,1]$ denotes the class of real valued differentiable functions defined on $[0,1]$, whose derivatives are of bounded variation on $[0,1]$, which can be written as

$$
f(x)=f(0)+\int_{0}^{x} \Psi(t) d t, \quad x \in[0,1]
$$

where $\Psi \in B V[0,1]$. In this sense it is justified to call this class of functions with derivatives of bounded variation and will be denoted as equivalently

$$
D B V[0,1]=\left\{f: f^{\prime} \in B V[0,1]\right\} .
$$

The main result of this paper is the following assertion.

Theorem. Let $\alpha \geq 1$ and $f$ be a function with derivatives of bounded variation on $[0,1]$. If $f^{\prime}$ has a discontinuity of the first kind in $x \in(0,1)$, then for each $\lambda>2$ and $\varepsilon>0$, there is an integer $N(x, \lambda)$ such that for all $n \geq N(x, \lambda)$ we have

$$
\begin{aligned}
\left|M_{n, \alpha}(f ; x)-f(x)\right| \leq & \frac{\alpha}{\alpha+1}\left|f^{\prime}(x+)-f^{\prime}(x-)\right| \sqrt{\frac{\alpha \lambda(x+\varepsilon)(1-x)^{2}}{n}} \\
& +\frac{\alpha \lambda(x+\varepsilon)(1-x)}{n-1} \frac{1}{\alpha+1}\left|f^{\prime}(x+)+\alpha f^{\prime}(x-)\right| \\
& +\frac{1}{\sqrt{n}} \bigvee_{x-\frac{x}{\sqrt{n}}}^{x+\frac{1-x}{\sqrt{n}}}\left(f_{x}^{\prime}\right)+\frac{\alpha \lambda(x+\varepsilon)+x}{n x} \sum_{k=1}^{[\sqrt{n}] x+\frac{1-x}{k}} \bigvee_{x-\frac{x}{k}}\left(f_{x}^{\prime}\right),
\end{aligned}
$$


where

$$
f_{x}(t)= \begin{cases}f(t)-f(x+), & x<t \leq 1 \\ 0, & t=x \\ f(t)-f(x-), & 0 \leq t<x\end{cases}
$$

and $\bigvee_{a}^{b}\left(f_{x}^{\prime}\right)$ is the total variation of $f_{x}^{\prime}$ on $[a, b]$.

\section{Auxiliary Results}

In this section we give certain results, which are necessary to prove our main theorem.

Lemma 1 ([9]). For $s \in \mathbb{N}^{0}$ (the set of nonnegative integers), if we define

$$
M_{n, 1}\left((t-x)^{s} ; x\right)=\sum_{k=0}^{\infty} m_{n, k}(x) \int_{0}^{1} b_{n, k}(t)(t-x)^{s} d t
$$

then

$$
\left|M_{n, 1}((t-x) ; x)\right|=\frac{x(1-x)}{n-1}
$$

and

$$
M_{n, 1}\left((t-x)^{2} ; x\right) \leq \frac{4 x}{n-1}+\frac{2(1-x)^{2}}{(n-1)(n-2)} .
$$

In particular, given $\lambda>2$ and $\varepsilon>0$, there is an integer $N(x, \lambda)$ such that for all $n \geq N(x, \lambda)$ and $x \in[0,1]$,

$$
\left|M_{n, 1}((t-x) ; x)\right| \leq \frac{\lambda(x+\varepsilon)(1-x)}{n-1}
$$

and

$$
M_{n, 1}\left((t-x)^{2} ; x\right) \leq \frac{\lambda(x+\varepsilon)(1-x)^{2}}{n} .
$$

Remark 1 ([15]). For all $n, k \in N$, there holds $Q_{n, k}^{\alpha}(x) \leq \alpha m_{n, k}(x), x \in[0,1]$.

Define

$$
K_{n, \alpha}(x, t)=\sum_{k=0}^{\infty} Q_{n, k}^{\alpha}(x) b_{n, k}(t)
$$

and

$$
\lambda_{n, \alpha}(x, t)=\int_{0}^{t} K_{n, \alpha}(x, u) d u
$$

Note that

$$
\lambda_{n, \alpha}(x, 1)=\int_{0}^{1} K_{n, \alpha}(x, u) d u=1 .
$$


Lemma $2([9])$. For each $\lambda>2$ and $\varepsilon>0$, there is an integer $N(x, \lambda)$ such that, for all $n \geq N(x, \lambda)$ and $x \in(0,1)$,

$$
\begin{aligned}
\lambda_{n, \alpha}(x, y) \leq \alpha \frac{\lambda(x+\varepsilon)(1-x)^{2}}{n(x-y)^{2}}, & 0 \leq y<x, \\
1-\lambda_{n, \alpha}(x, z) \leq \alpha \frac{\lambda(x+\varepsilon)(1-x)^{2}}{n(z-x)^{2}}, & x<z \leq 1 .
\end{aligned}
$$

Remark 2. From Cauchy-Schwarz-Bunyakowsky inequality, we get from (9)

$$
M_{n, \alpha}(|t-x| ; x) \leq\left(M_{n, \alpha}\left((t-x)^{2} ; x\right)\right)^{\frac{1}{2}} \leq \sqrt{\alpha \frac{\lambda(x+\varepsilon)(1-x)^{2}}{n}} .
$$

\section{Proof of The Main Result}

Now, we can prove the theorem.

Proof of Theorem. According to (5) and equation (10), we can write the difference between $M_{n, \alpha}(f ; x)$ and $f(x)$ as follows:

$$
\begin{aligned}
M_{n, \alpha}(f ; x)-f(x) & =\sum_{k=0}^{\infty} Q_{n, k}^{\alpha}(x) \int_{0}^{1} b_{n, k}(t) f(t) d t-f(x) \\
& =\int_{0}^{1}[f(t)-f(x)] K_{n, \alpha}(x, t) d t .
\end{aligned}
$$

Since $f(t) \in D B V[0,1]$, we can rewrite equation (14) as

$$
\begin{aligned}
M_{n, \alpha}(f ; x) & -f(x)=\int_{0}^{x}[f(t)-f(x)] K_{n, \alpha}(x, t) d t+\int_{x}^{1}[f(t)-f(x)] K_{n, \alpha}(x, t) d t \\
& =-\int_{0}^{x}\left[\int_{t}^{x} f^{\prime}(u) d u\right] K_{n, \alpha}(x, t) d t+\int_{x}^{1}\left[\int_{x}^{t} f^{\prime}(u) d u\right] K_{n, \alpha}(x, t) d t \\
& =-I_{1}(x)+I_{2}(x),
\end{aligned}
$$

where

$$
I_{1}(x):=\int_{0}^{x}\left[\int_{t}^{x} f^{\prime}(u) d u\right] K_{n, \alpha}(x, t) d t
$$

and

$$
I_{2}(x):=\int_{x}^{1}\left[\int_{x}^{t} f^{\prime}(u) d u\right] K_{n, \alpha}(x, t) d t
$$


From (7), for any $f(t) \in D B V[0,1]$, we decompose $f^{\prime}(t)$ into four parts as

$$
\begin{aligned}
f^{\prime}(t) & =\frac{1}{\alpha+1}\left(f^{\prime}(x+)+\alpha f^{\prime}(x-)\right)+f_{x}^{\prime}(t)+\left(\frac{f^{\prime}(x+)-f^{\prime}(x-)}{2}\right) \\
& \times\left(\operatorname{sgn}(t-x)+\frac{\alpha-1}{\alpha+1}\right)+\delta_{x}(t)\left(f^{\prime}(x)-\frac{f^{\prime}(x+)+f^{\prime}(x-)}{2}\right),
\end{aligned}
$$

where

$$
\delta_{x}(t)= \begin{cases}1, & x=t \\ 0, & x \neq t\end{cases}
$$

If we use (17) in (15) and (16), we have the following expressions.

$$
\begin{aligned}
& I_{1}(x)=\int_{0}^{x}\left[\int _ { t } ^ { x } \left\{\frac{1}{\alpha+1}\left(f^{\prime}(x+)+\alpha f^{\prime}(x-)\right)+f_{x}^{\prime}(u)\right.\right. \\
& +\left(\frac{f^{\prime}(x+)-f^{\prime}(x-)}{2}\right)\left(\operatorname{sgn}(u-x)+\frac{\alpha-1}{\alpha+1}\right) \\
& \left.\left.\quad+\delta_{x}(t)\left(f^{\prime}(x)-\frac{f^{\prime}(x+)+f^{\prime}(x-)}{2}\right)\right\} d u\right] K_{n, \alpha}(x, t) d t
\end{aligned}
$$

and

$$
\begin{aligned}
I_{2}(x)=\int_{x}^{1}\left[\int _ { x } ^ { t } \left\{\frac{1}{\alpha+1}\left(f^{\prime}(x+)+\alpha f^{\prime}(x-)\right)+f_{x}^{\prime}(u)\right.\right. \\
+\left(\frac{f^{\prime}(x+)-f^{\prime}(x-)}{2}\right)\left(\operatorname{sgn}(u-x)+\frac{\alpha-1}{\alpha+1}\right) \\
\left.\left.\quad+\delta_{x}(t)\left[f^{\prime}(x)-\frac{\left.f^{\prime}(x+)+f^{\prime}(x-)\right)}{2}\right\}\right\} d u\right] K_{n, \alpha}(x, t) d t .
\end{aligned}
$$

Firstly, we evaluate $I_{1}(x)$.

By (18), it is obvious that $\int_{x}^{t} \delta_{x}(u) d u=0$. We have

$$
\begin{aligned}
I_{1}(x)= & \frac{1}{\alpha+1}\left[f^{\prime}(x+)+\alpha f^{\prime}(x-)\right] \int_{0}^{x}(x-t) K_{n, \alpha}(x, t) d t \\
& +\frac{f^{\prime}(x+)-f^{\prime}(x-)}{2} \int_{0}^{x}\left[-1+\frac{\alpha-1}{\alpha+1}\right](x-t) K_{n, \alpha}(x, t) d t \\
& +\int_{0}^{x}\left[\int_{t}^{x} f_{x}^{\prime}(u) d u\right] K_{n, \alpha}(x, t) d t .
\end{aligned}
$$


Using similar method, for evaluating $I_{2}(x)$, we find that

$$
\begin{aligned}
I_{2}(x)= & \frac{1}{\alpha+1}\left[f^{\prime}(x+)+\alpha f^{\prime}(x-)\right] \int_{x}^{1}(t-x) K_{n, \alpha}(x, t) d t \\
& +\frac{f^{\prime}(x+)-f^{\prime}(x-)}{2} \int_{x}^{1}\left[1+\frac{\alpha-1}{\alpha+1}\right](t-x) K_{n, \alpha}(x, t) d t \\
& +\int_{x}^{1}\left[\int_{x}^{t} f_{x}^{\prime}(u) d u\right] K_{n, \alpha}(x, t) d t .
\end{aligned}
$$

Since $\alpha \geq 1$, from equations (19) and (20), we obtain an estimate for the difference between $M_{n, \alpha}(f ; x)$ and $f(x)$ as follows;

$$
\begin{aligned}
\left|M_{n, \alpha}(f ; x)-f(x)\right| \leq & \frac{1}{\alpha+1}\left|f^{\prime}(x+)+\alpha f^{\prime}(x-)\right|\left|\int_{0}^{1}(t-x) K_{n, \alpha}(x, t) d t\right| \\
& +\frac{\alpha}{\alpha+1}\left|f^{\prime}(x+)-f^{\prime}(x-)\right|\left|\int_{0}^{1}\right| t-x\left|K_{n, \alpha}(x, t) d t\right| \\
& +\left|-\int_{0}^{x}\left[\int_{t}^{x} f_{x}^{\prime}(u) d u\right] K_{n, \alpha}(x, t) d t\right| \\
& +\left|\int_{x}^{1}\left[\int_{x}^{t} f_{x}^{\prime}(u) d u\right] K_{n, \alpha}(x, t) d t\right| .
\end{aligned}
$$

On the other hand, since

$$
\int_{0}^{1}|t-x| K_{n, \alpha}(x, t) d t=M_{n, \alpha}(|t-x| ; x)
$$

and

$$
\int_{0}^{1}(t-x) K_{n, \alpha}(x, t) d t=M_{n, \alpha}(t-x ; x),
$$

then using (22) and (23) in (21), we obtain

$$
\begin{aligned}
\left|M_{n, \alpha}(f ; x)-f(x)\right| \leq & \frac{1}{\alpha+1}\left|f^{\prime}(x+)+\alpha f^{\prime}(x-)\right|\left|M_{n, \alpha}(t-x ; x)\right| \\
& +\frac{\alpha}{\alpha+1}\left|f^{\prime}(x+)-f^{\prime}(x-)\right|\left|M_{n, \alpha}(|t-x| ; x)\right| \\
& +\left|-\int_{0}^{x}\left[\int_{t}^{x} f_{x}^{\prime}(u) d u\right] K_{n, \alpha}(x, t) d t\right|
\end{aligned}
$$




$$
+\left|\int_{x}^{1}\left[\int_{x}^{t} f_{x}^{\prime}(u) d u\right] K_{n, \alpha}(x, t) d t\right| .
$$

From the definition of $\lambda_{n, \alpha}(x, t)$, we write

$$
\int_{0}^{x}\left[\int_{t}^{x} f_{x}^{\prime}(u) d u\right] K_{n, \alpha}(x, t) d t=\int_{0}^{x}\left[\int_{t}^{x} f_{x}^{\prime}(u) d u\right] \frac{\partial}{\partial t} \lambda_{n, \alpha}(x, t) d t .
$$

Using integration by parts in the right-hand side of (25), we obtain

$$
\int_{0}^{x}\left[\int_{t}^{x} f_{x}^{\prime}(u) d u\right] \frac{\partial}{\partial t} \lambda_{n, \alpha}(x, t) d t=\int_{0}^{x} f_{x}^{\prime}(t) \lambda_{n, \alpha}(x, t) d t .
$$

Thus

$$
\left|-\int_{0}^{x}\left[\int_{t}^{x} f_{x}^{\prime}(u) d u\right] K_{n, \alpha}(x, t) d t\right| \leq \int_{0}^{x}\left|f_{x}^{\prime}(t)\right| \lambda_{n, \alpha}(x, t) d t
$$

and

$$
\begin{aligned}
\left|-\int_{0}^{x}\left[\int_{t}^{x} f_{x}^{\prime}(u) d u\right] K_{n, \alpha}(x, t) d t\right| \leq & \int_{0}^{x-\frac{x}{\sqrt{n}}}\left|f_{x}^{\prime}(t)\right| \lambda_{n, \alpha}(x, t) d t \\
& +\int_{x-\frac{x}{\sqrt{n}}}^{x}\left|f_{x}^{\prime}(t)\right| \lambda_{n, \alpha}(x, t) d t .
\end{aligned}
$$

Since $f_{x}^{\prime}(x)=0$ and $\lambda_{n, \alpha}(x, t) \leq 1$,

$$
\begin{aligned}
\int_{x-\frac{x}{\sqrt{n}}}^{x}\left|f_{x}^{\prime}(t)\right| \lambda_{n, \alpha}(x, t) d t & =\int_{x-\frac{x}{\sqrt{n}}}^{x}\left|f_{x}^{\prime}(t)-f_{x}^{\prime}(x)\right| \lambda_{n, \alpha}(x, t) d t \\
& \leq \int_{x-\frac{x}{\sqrt{n}}}^{x} \bigvee_{t}^{x}\left(f_{x}^{\prime}\right) d t .
\end{aligned}
$$

Besides from (11), we have

$$
\begin{aligned}
\int_{0}^{x-\frac{x}{\sqrt{n}}}\left|f_{x}^{\prime}(t)\right| \lambda_{n, \alpha}(x, t) d t & \leq \frac{\alpha \lambda(x+\varepsilon)(1-x)^{2}}{n} \int_{0}^{x-\frac{x}{\sqrt{n}}}\left|f_{x}^{\prime}(t)\right| \frac{d t}{(x-t)^{2}} \\
& \leq \frac{\alpha \lambda(x+\varepsilon)(1-x)^{2}}{n} \int_{0}^{x-\frac{x}{\sqrt{n}}} \bigvee_{t}^{x}\left(f_{x}^{\prime}\right) \frac{d t}{(x-t)^{2}} .
\end{aligned}
$$


Make the change of variables $t=x-\frac{x}{u}$, then

$$
\int_{0}^{x-\frac{x}{\sqrt{n}}} \bigvee_{t}^{x}\left(f_{x}^{\prime}\right) \frac{d t}{(x-t)^{2}}=\int_{1}^{\sqrt{n}} \bigvee_{x-\frac{x}{u}}^{x}\left(f_{x}^{\prime}\right) \frac{\left(\frac{x}{u^{2}}\right) d u}{\left(-\frac{x}{u}\right)^{2}}=\frac{1}{x} \int_{1}^{\sqrt{n}} \bigvee_{x-\frac{x}{u}}^{x}\left(f_{x}^{\prime}\right) d u=\frac{1}{x} \sum_{k=1}^{[\sqrt{n}]} \bigvee_{x-\frac{x}{k}}^{x}\left(f_{x}^{\prime}\right)
$$

and

$$
\int_{x-\frac{x}{\sqrt{n}}}^{x} \bigvee_{t}^{x}\left(f_{x}^{\prime}\right) d t \leq \bigvee_{x-\frac{x}{\sqrt{n}}}^{x}\left(f_{x}^{\prime}\right) \int_{x-\frac{x}{\sqrt{n}}}^{x} d t=\frac{x}{\sqrt{n}} \bigvee_{x-\frac{x}{\sqrt{n}}}^{x}\left(f_{x}^{\prime}\right)
$$

Consequently

$$
\begin{aligned}
\left|-\int_{0}^{x}\left[\int_{t}^{x} f_{x}^{\prime}(u) d u\right] K_{n, \alpha}(x, t) d t\right| \leq & \frac{\alpha \lambda(x+\varepsilon)(1-x)^{2}}{n x} \sum_{k=1}^{[\sqrt{n}]} \bigvee_{x-\frac{x}{k}}^{x}\left(f_{x}^{\prime}\right) \\
& +\frac{x}{\sqrt{n}} \bigvee_{x-\frac{x}{\sqrt{n}}}^{x}\left(f_{x}^{\prime}\right) .
\end{aligned}
$$

By the same way, from (12) we obtain

$$
\begin{aligned}
\left|\int_{x}^{1}\left[\int_{x}^{t} f_{x}^{\prime}(u) d u\right] K_{n, \alpha}(x, t) d t\right| \leq & \frac{\alpha \lambda(x+\varepsilon)(1-x)}{n} \sum_{k=1}^{[\sqrt{n}]} \bigvee_{x}^{x+\frac{1-x}{k}}\left(f_{x}^{\prime}\right) \\
& +\frac{1-x}{\sqrt{n}} \bigvee_{x}^{x+\frac{1-x}{\sqrt{n}}}\left(f_{x}^{\prime}\right) .
\end{aligned}
$$

Combining (8), (13), (26) and (27) in (24), we get (6).

Thus the proof is completed.

\section{REFERENCES}

1. P. BÉzIER, Numerical control. Mathematics and applications. (Translated from the French) Wiley Series in Computing. John Wiley \& Sons, London, etc., 1972.

2. R. BoJanić and F. H. ChÊNG, Rate of convergence of Bernstein polynomials for functions with derivatives of bounded variation. J. Math. Anal. Appl. 141(1989), No. 1, $136-151$.

3. R. Bojanić and F. H. ChÊng, Rate of convergence of Hermite-Fejér polynomials for functions with derivatives of bounded variation. Acta Math. Hungar. 59(1992), No. 1-2, $91-102$.

4. R. Bojanic and M. K. Khan, Rate of convergence of some operators of functions with derivatives of bounded variation. Atti Sem. Mat. Fis. Univ. Modena 39(1991), No. 2, 495-512.

5. S. S. Guo, Degree of approximation to functions of bounded variation by certain operators. Approx. Theory Appl. 4(1988), No. 2, 9-18.

6. S. S. Guo, On the rate of convergence of the integrated Meyer-König and Zeller operators for functions of bounded variation. J. Approx. Theory 56(1989), No. 3, 245-255. 
7. V. Gupta, A sharp estimate on the degree of approximation to functions of bounded variation by certain operators. Approx. Theory Appl. (N.S.) 11(1995), No. 3, 106-107.

8. V. Gupta, On a new type of Meyer-Konig and Zeller operators. J. Inequal. Pure Appl. Math. 3(2002), No. 4, Article 57, 10 pp. (electronic).

9. V. Gupta, On bounded variation functions by general MKZD operators. Acta Math. Sin. (Engl. Ser.) 23(2007), No. 8, 1457-1462.

10. V. Gupta, U. Abel, and M. Ivan, Rate of convergence of beta operators of second kind for functions with derivatives of bounded variation. Int. J. Math. Math. Sci. 2005, No. 23, 3827-3833.

11. V. Gupta and A. Ahmad, An improved estimate on the degree of approximation to functions of bounded variation by certain operators. Rev. Colombiana Mat. 29(1995), No. 2, 119-126.

12. V. Gupta, V. Vasishtha, and M. K. Gupta, Rate of convergence of summationintegral type operators with derivatives of bounded variation. J. Inequal. Pure Appl. Math. 4(2003), No. 2, Article 34, 8 pp. (electronic).

13. W. Meyer-König and K. Zeller, Bernsteinsche Potenzreihen. Studia Math. 19(1960), 89-94.

14. P. PyCh-TABerska, Pointwise approximation of absolutely continuous functions by certain linear operators. Dedicated to Roman Taberski on the occasion of his 70th birthday. Funct. Approx. Comment. Math. 25(1997), 67-76.

15. X. M. ZENG, Rates of approximation of bounded variation functions by two generalized Meyer-König and Zeller type operators. Comput. Math. Appl. 39(2000), No. 9-10, 1-13.

(Received 30.04.2006)

Authors' addresses:

V. Gupta

School of Applied Sciences

Netaji Subhas Institute of Technology

Sector 3 Dwarka, New Delhi-110075

India

E-mail: vijaygupta2001@hotmail.com

H. Karsli

Ankara University

Faculty of Sciences

Department of Mathematics

06100 Tandogan-Ankara

Turkey

E-mail: karsli@science.ankara.edu.tr 